\title{
Neural mechanisms of subclinical depressive symptoms in women: a pilot functional brain imaging study
}

\author{
Jennifer N Felder ${ }^{1,5^{*}}$, Moria J Smoski ${ }^{2}$, Rachel V Kozink ${ }^{2}$, Brett Froeliger ${ }^{2}$, Joseph McClernon ${ }^{2,4}$, Joshua Bizzell ${ }^{1,3,4}$, \\ Christopher Petty ${ }^{4}$ and Gabriel S Dichter ${ }^{1,2,3,4}$
}

\begin{abstract}
Background: Studies of individuals who do not meet criteria for major depressive disorder (MDD) but with subclinical levels of depressive symptoms may aid in the identification of neurofunctional abnormalities that possibly precede and predict the development of MDD. The purpose of this study was to evaluate relations between subclinical levels of depressive symptoms and neural activation patterns during tasks previously shown to differentiate individuals with and without MDD.

Methods: Functional magnetic resonance imaging (fMRI) was used to assess neural activations during active emotion regulation, a resting state scan, and reward processing. Participants were twelve females with a range of depressive symptoms who did not meet criteria for MDD.

Results: Increased depressive symptom severity predicted (1) decreased left midfrontal gyrus activation during reappraisal of sad stimuli; (2) increased right midfrontal gyrus activation during distraction from sad stimuli; (3) increased functional connectivity between a precuneus seed region and left orbitofrontal cortex during a resting state scan; and (4) increased paracingulate activation during non-win outcomes during a reward-processing task.

Conclusions: These pilot data shed light on relations between subclinical levels of depressive symptoms in the absence of a formal MDD diagnosis and neural activation patterns. Future studies will be needed to test the utility of these activation patterns for predicting MDD onset in at-risk samples.
\end{abstract}

Keywords: FMRI, Depression symptoms, Emotion regulation, Resting state, Reward

\section{Background}

Despite a large body of research addressing impaired neural functioning in major depressive disorder (MDD), relatively little is known about the neurofunctional characteristics of individuals without MDD but with subclinical levels of depressive symptoms. Studies of individuals who do not meet criteria for MDD but with subclinical levels of depressive symptoms may yield a number of insights: first, they may suggest potential neurobiologic markers of those at risk for MDD [1,2];

\footnotetext{
* Correspondence: Jennifer.Felder@colorado.edu

'Carolina Institute for Developmental Disabilities, University of North Carolina at Chapel Hill School of Medicine, CB\# 3366, 101 Manning Drive, Chapel Hill, NC 27599-7160, USA

${ }^{5}$ Department of Psychology and Neuroscience, University of Colorado Boulder, UCB 345, Boulder, CO 80309-0345, USA

Full list of author information is available at the end of the article
}

second, they may shed light on hereditary and environmental influences on depressive temperament [3]; and third, they may suggest avenues of inquiry regarding the neurobiology of MDD risk and resilience, and thereby inform treatment and preventative intervention approaches $[4,5]$. The goal of the present pilot study was to investigate linkages between regional brain activation patterns and subclinical depressive symptoms while participants were engaged in emotion regulation, resting state, and reward processing paradigms that have previously been shown to differentiate MDD and nondepressed samples. Below we outline the rationale for selecting each of these three paradigms.

Deficits in emotion regulation (ER) are thought to be central to the core features of MDD [6]. Human and preclinical studies suggest that the prefrontal cortex

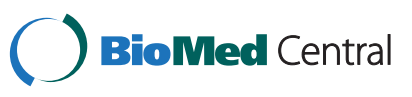

(c) 2012 Felder et al.; licensee BioMed Central Ltd. This is an Open Access article distributed under the terms of the Creative Commons Attribution License (http://creativecommons.org/licenses/by/2.0), which permits unrestricted use, distribution, and reproduction in any medium, provided the original work is properly cited. 
influences activity in the limbic system in a top-down manner $[7,8]$, and numerous investigations have demonstrated abnormal recruitment of prefrontal cortical regions while individuals with MDD actively regulate responses to emotional stimuli $[9,10]$. For example, Johnstone and colleagues [6] reported that when instructed to reappraise negatively valenced images, individuals with MDD demonstrated impaired prefrontal cortical inhibition of limbic regions as well as a divergent pattern of covariation between ventromedial prefrontal cortex and amygdala activity. Similarly, Beauregard and colleagues [11] reported right anterior cingulate and insular cortex hyperactivation while individuals with MDD down-regulated their emotional responses to sad images. Finally, Kanske and colleagues [12] reported anterior cingulate and lateral orbitofrontal cortex hyperactivation during emotion regulation in individuals with remitted MDD , supporting the framework that altered neural mechanisms of emotion regulation may indeed represent not only a state marker of MDD illness, but potentially a trait marker of MDD risk as well.

Second, we investigated resting state functional brain connectivity. A mostly midline network of correlated intrinsic brain activity is active during rest and deactive during goal-directed tasks $[13,14]$. This network has been called both the "baseline state" and the "default mode network (DMN)" $[15,16]$. The DMN regulates self-referential activities, including evaluating the salience of internal and external cues, remembering the past, and planning the future [13,17]. Individuals with MDD are characterized by altered patterns of DMN activity during emotion processing tasks as well as at rest. Sheline and colleagues [18] reported increased activity in MDD within DMN regions (i.e., ventromedial prefrontal cortex, anterior cingulate, and lateral parietal and temporal cortices), suggesting that MDD is characterized by a failure to down-regulate DMN activity during emotion processing. Another study by the same research group [19] reported increased intrinsic connectivity in MDD between a precuneus seed region and dorsolateral prefrontal cortex (the so-called "dorsal nexus" for overconnected brain regions in MDD) (see also [16] and [20]). Given the centrality of the DMN for selfreferential activities, abnormal DMN activity in MDD has been suggested to reflect excessive self-focus accompanied by a decreased ability to attend to cognitive tasks [19].

Finally, we measured frontostriatal responses during a reward processing task because multiple studies have reported altered neural mechanisms of reward processing in MDD (for a review, see [21]). For example, Smoski and colleagues [22] reported that individuals with MDD demonstrated orbital frontal cortex hyperactivity during reward selection that predicted depressive symptom severity and caudate nucleus hypoactivation during reward anticipation. Pizzagalli and colleagues [23] found that individuals with MDD showed decreased putamen responses during reward anticipation and decreased nucleus accumbens and caudate nucleus responses to monetary gains. Finally, Knutson and colleagues [24] reported increased anterior cingulate activation during reward anticipation in MDD. Taken together, these studies highlight not only that MDD is characterized by altered neural activation patterns during reward processing, but that patterns of brain activation differences in MDD are contingent on the temporal phase of the reward response. For this reason, we used a task that allowed for the dissociation of responses during reward selection, reward anticipation, and reward outcome phases of reward responding.

In summary, the purpose of the present study was to extend the literature addressing altered neural mechanisms of emotion regulation, DMN connectivity, and reward processing in MDD by examining the covariation between levels of depressive symptoms and patterns of brain activation in individuals with subclinical levels of depressive symptoms but without MDD diagnoses. Predictions were informed by the extant MDD literature reviewed above $[6,19,22]$ : we hypothesized relations between subclinical depressive symptom severity and (1) lateral prefrontal cortex activity during ER; (2) precuneus-dorsolateral prefrontal cortex connectivity during the resting state scan; (3) lateral prefrontal cortex activity during reward selection; (4) striatal activity during reward anticipation; and (5) medial prefrontal cortex activity during reward outcomes. Given the higher rates of MDD in women [25] and because gender moderates responses to emotional images [26,27], this pilot study examined only females, a strategy that is consistent with other neuroimaging studies examining MDD [28], depression risk [29] and depressive traits [30].

\section{Methods}

\section{Participants}

Participants consented to protocols approved by the local Human Investigations Committees at both the University of North Carolina Chapel Hill and Duke University Medical Center and were recruited from a database maintained at the Duke-UNC Brain Imaging and Analysis Center and by flyers posted in campus and medical center locations. To identify individuals with moderate but not severe depression symptoms, interested respondents first completed a modified the Beck Depression Inventory-II [BDI, 31] via a secure web survey. Next, potential participants completed an in-person screening session that included the full BDI and administration of the Structured Clinical Interview for DSM-IV, Patient 
Version, with Psychotic Screen (SCID) [32]. The final sample was recruited based on a stratification strategy to recruit equal numbers of individuals with low (i.e., 0-9), medium (i.e., 10-18), and high (i.e., 19+) BDI scores. Exclusion criteria included: 1) current anxiety, substance abuse, or mood disorder beyond dysthymia (as assessed by the SCID), 2) history of mania, 3) currently taking psychoactive medication, 4) magnetic resonance imaging contraindicated (e.g., metal in body), 5) history of neurological injury or disease, and 6) pregnancy. The final sample was twelve females (mean age: $23.3 \pm 4.3 ; 3$ African American; 3 Asian, and six Caucasian) who participated in the scan session.

\section{Pre-scan emotion regulation training session}

Participants completed a training session prior to the scan day at which they were taught strategies to distract themselves and reappraise their interpretations while viewing sad stimuli. For the distract condition, participants were trained to think of something positive that was unrelated to what was depicted in the images, such as a fond memory, family member, or an anticipated event. For the reappraise condition, participants were trained to either give the story suggested in the picture a happy ending or alternate meaning, or to tell themselves that no one close to them was affected by the depicted situation. As a control strategy, participants were instructed to experience the image as they normally would without trying to increase or decrease their emotional response (the "view" condition).

\section{Scan day procedure}

On the day of the scan, participants reviewed task instructions and completed the BDI again. During scanning, participants completed the three paradigms described below in a single scan session. Due to technical problems, three participants returned to complete the emotion regulation task at a separate imaging session, at which time the BDI was readministered. Analyses included only BDI scores obtained on the day that each task was completed. The mean BDI scores in final analyses were 11.33 ( $\mathrm{SD}=10.21$, Range $=0$ to 26 , Skewness $=0.10)$ on the day of the resting state and reward processing scans, and $9.92(\mathrm{SD}=9.02$, Range $=0$ to 24 , Skewness $=0.29$ ) on the day of the ER scan.

\section{Emotion Regulation (ER) task}

The ER task consisted of four 4-minute 24-second runs with nine sad trials and three neutral trials each (see the top of Figure 1). The stimuli used in the task are described elsewhere [33,34]. During each 22-second trial, participants first fixated for 6 seconds and then saw a sad or neutral image for 11 seconds with an auditory instruction presented 3-6 seconds after image onset.
Sad images were presented on $75 \%$ of trials and were accompanied by an instruction to "reappraise," "distract," or "view" the image. Neutral images, on the other $25 \%$ of trials, were accompanied by an instruction to "view" the image. Following image offset, participants reported their ER success on a 4-point scale anchored by "not successful" and "very successful," or they indicated they felt "no emotion" (trials with "no emotion" were not included in analyses).

\section{Resting state scan}

Participants were instructed to close their eyes and relax but not fall asleep for the duration of the 6-minute resting state scan.

\section{Wheelof Fortune (WOF) reward processing task}

The WOF is described in greater detail elsewhere [35]. Briefly, three 12-minute runs with 46 trials each were composed of selection, anticipation, and outcome phases (see the bottom of Figure 1). During the selection phase (3 seconds), participants viewed a segmented wheel of fortune and indicated their selection. During the anticipation phase (3.5-7.5 seconds), participants rated how sure they were of winning on a scale of 1 to 6 (anchored by "unsure" and "sure"). During the outcome phase (4 seconds), participants viewed the amount won (" $\$ 0$ " for not winning), the total cumulative amount won in the run, and rated how they felt on a pictorial Likert scale. Responses were contrasted with a control condition that lacked monetary stimuli but required active responding.

\section{Imaging methods}

Scanning was performed on a GE 3 Tesla Signa Excite HD scanner with $50-\mathrm{mT} / \mathrm{m}$ gradients (General Electric, Waukesha, Wisconsin, USA). Head movement was restricted by foam cushions. An eight-channel head coil was used for parallel imaging. A high resolution T1weighted anatomical image was acquired using a 3D fast SPGR pulse sequence $(68$ slices, $T R=500 \mathrm{~ms}$; $\mathrm{TE}=20 \mathrm{~ms} ; \mathrm{FOV}=24 \mathrm{~cm}$; image matrix $=256^{2}$; voxel size $=0.9375 \times 0.9375 \times 1.9 \mathrm{~mm}$ ) aligned in the near axial plane defined by the anterior and posterior commissures. Whole brain functional images consisting of 30 slices parallel to the AC-PC plane were collected using a BOLD-sensitive gradient-echo sequence with spiral-in k-space sampling and SENSE encoding to take advantage of the 8-channel coil (TE: $30 \mathrm{~ms}$; FOV: $25.6 \mathrm{~cm}$; isotropic voxel size: $4 \mathrm{~mm}^{3}$; SENSE factor $=2$ ). Functional imaging sessions began with four discarded RF excitations to allow for steady state equilibrium. TR's were $1500 \mathrm{~ms}$ for the ER run and $2000 \mathrm{~ms}$ for the WoF and resting state runs. Images were presented using 


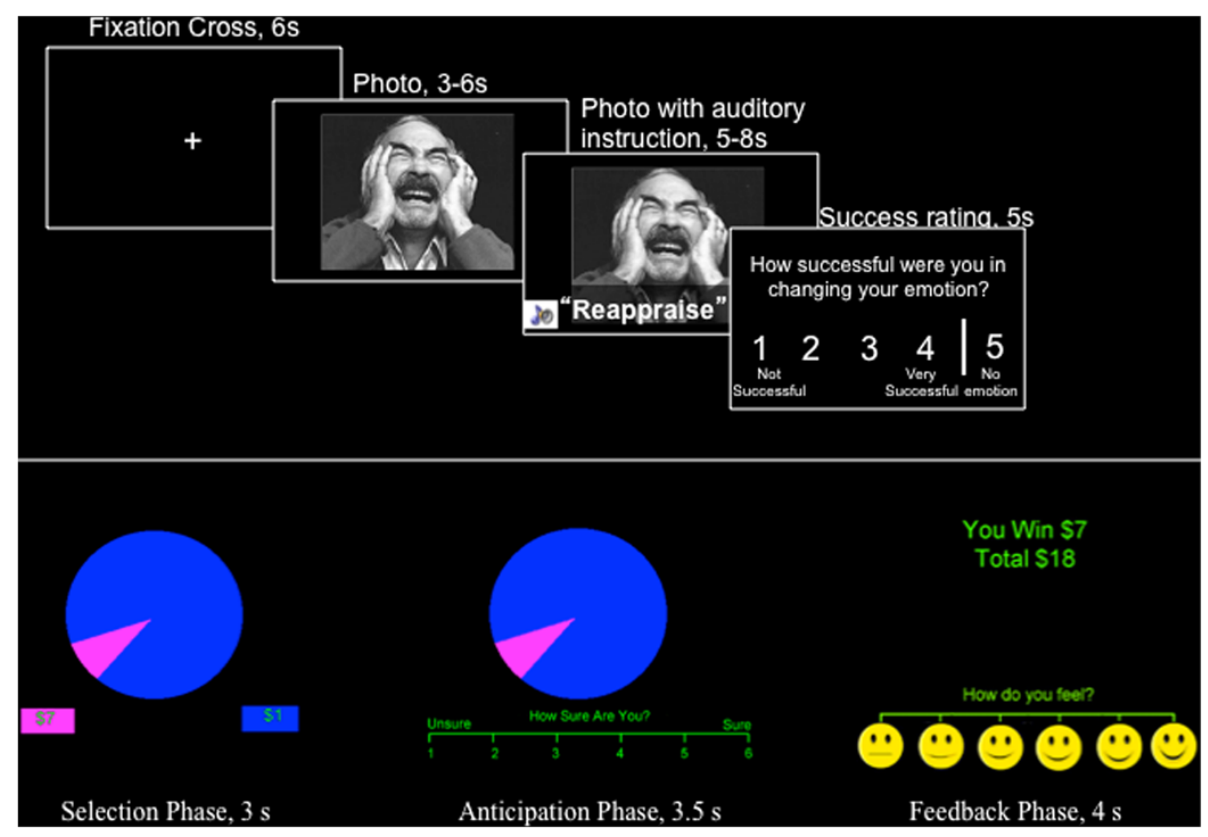

Figure 1 Top: Depiction of the emotion regulation task, showing durations of each phase; Bottom: Depiction of the Wheel of Fortune task; the area of the slices matched the likelihood of winning explicit amounts of money demoted below the wheel in squares of matching colors.

ePrime (Psychology Software Tools Inc., Pittsburgh, PA) for the WOF task, and with CIGAL [36] for the ER task.

\section{Imaging data analysis}

Functional data were preprocessed using FEAT 5.92 within FSL 4.0.4 (Oxford Centre for Functional Magnetic Resonance Imaging of the Brain (FMRIB), Oxford University, U.K.). Preprocessing was applied in the following steps: (i) brain extraction for non-brain removal on the T1-weighted image [37], (ii) motion correction using MCFLIRT [38], (iii) spatial smoothing using a Gaussian kernel of FWHM $5 \mathrm{~mm}$, (iv) mean-based intensity normalization of all volumes by the same factor, and (v) high-pass filtering (60 s) [39]. Functional images were co-registered to the brain-extracted T1weighted image in native space, and the anatomical image was normalized to Montreal Neurological Institute (MNI) standard stereotaxic space. The same transformation matrices used for anatomical-to-standard transformations were then used for functional-tostandard space transformations of co-registered functional images. All registrations were carried out using an intermodal registration tool $[37,39]$. Voxel-wise temporal autocorrelation was estimated and corrected using FMRIB's Improved Linear Model [40].

For ER and WOF data, onset times of events were used to model a signal response containing a regressor for each response type, which was convolved with a double-gamma function to model the predicted hemodynamic response. A general linear model (GLM) approach generated whole brain images of parameter estimates and variances, representing average signal change from baseline. The same high-pass filtering applied to the functional data was applied to the GLM. Group-wise activation images were calculated by a mixed effects analysis using Bayesian estimation techniques, FMRIB Local Analysis of Mixed Effects [FILM, 41]. Group-level models, with mean-centered BDI scores as regressors, were created by combining contrast maps from individual subjects. Finally, scatterplots of correlations between brain activation and BDI score are presented for illustrative purposes [42].

Analysis of resting state data used the following seedbased connectivity approach: (1) raw functional scans were temporally band-pass filtered $(0.1<f<.08)$; (2) as in [15], the mean signal intensity timecourses from voxels inside a spherical ROI (radius $=10 \mathrm{~mm}$ ) in the precuneus (MNI coordinates $0,-56,30$ ) were extracted for all participants in native space; (3) average signal intensity timecourses derived from the previous step were then used as unconvolved regressors in a GLM that included motion parameters, resulting in clusters significantly correlated with the timecourse of the seed region; (4) a group-level model was created by combining contrast maps from individual participants.

Given the importance of protecting against Type II errors in this exploratory pilot study, activation maps were thresholded at $Z>2.3$ with a spatial extent of 
at least ten voxels, consistent with published recommendations $[43,44]$ and our prior MDD studies [25,45-47].

\section{Results}

In-scanner emotion regulation success

There were no differences in self-reported ER success across the four ER conditions (Neutral-View, Sad-View, Sad-Distract, and Sad-Reappraise), $p$ 's $>0.80$. There was a significant inverse relation between BDI scores and success ratings only in the Sad-Reappraise condition, $r=-0.71, p<.01$, reflecting poorer self-reported ER in those with higher BDI scores.

\section{Emotion regulation imaging data}

\section{Sad-reappraise > Sad-view}

The top of Figure 2 depicts results during SadReappraise $>$ Sad-View (see Table 1). Areas that showed activation included the paracingulate gyrus ( $\mathrm{Z}$ Max = 4.36), right middle frontal gyrus $(Z M a x=3.07)$, and bilateral temporal gyrus ( $\mathrm{Z}$ Max $=4.14$ for left and 3.81 for right). BDI scores were inversely related to activation magnitudes in the left middle frontal gyrus ( $\mathrm{Z}$ Max $=$ $2.98)$ and right orbitofrontal cortex $(Z$ Max $=2.82)$ (see Table 2).
Table 1 Clusters showing significant activation during the Emotion Regulation task [Sad Reappraise > Sad View]

\begin{tabular}{|c|c|c|c|c|c|}
\hline \multirow[t]{2}{*}{ Region } & \multirow{2}{*}{$\begin{array}{c}\text { Size } \\
\left(\mathrm{mm}^{3}\right)\end{array}$} & \multirow{2}{*}{$\begin{array}{c}Z \\
\text { Max }\end{array}$} & \multicolumn{3}{|c|}{ MNI Coordinates } \\
\hline & & & $x$ & $\mathrm{Y}$ & Z \\
\hline Frontal Gyrus (Right, Middle) & 688 & 3.07 & 48 & 12 & 48 \\
\hline Frontal Gyrus (Left, Superior) & 280 & 2.83 & -18 & 8 & 68 \\
\hline Frontal Pole (Left) & 472 & 2.98 & -28 & -44 & -24 \\
\hline Hippocampus (Right) & 184 & 2.54 & 32 & -20 & -12 \\
\hline Intracalcarine Cortex (Left) & 176 & 2.56 & -18 & -68 & 4 \\
\hline $\begin{array}{l}\text { Occipital Cortex } \\
\text { (Left, Lateral, Inferior) }\end{array}$ & 896 & 2.92 & -42 & -70 & -20 \\
\hline Paracingulate Gyrus (Left) & 164248 & 4.36 & 0 & 50 & 12 \\
\hline Putamen (Right) & 240 & 2.92 & 22 & -2 & 8 \\
\hline \multicolumn{6}{|l|}{$\begin{array}{l}\text { Temporal Gyrus } \\
\text { (Middle, Posterior) }\end{array}$} \\
\hline Right & 8792 & 3.81 & -48 & -30 & -4 \\
\hline Left & 26600 & 4.14 & 50 & -28 & -6 \\
\hline
\end{tabular}

Clusters are $>10$ voxels with $Z>2.3$.

\section{Sad-Distract > Sad-View}

The bottom of Figure 2 depicts group-average results during Sad-Distract $>$ Sad-View (see also Table 3). Areas that showed activation included the cingulate gyrus $(Z$ $M a x=4.54)$, right supramarginal gyrus $(Z M a x=3.63)$, and right lateral occipital cortex $(\mathrm{Z}$ Max $=3.39)$. BDI

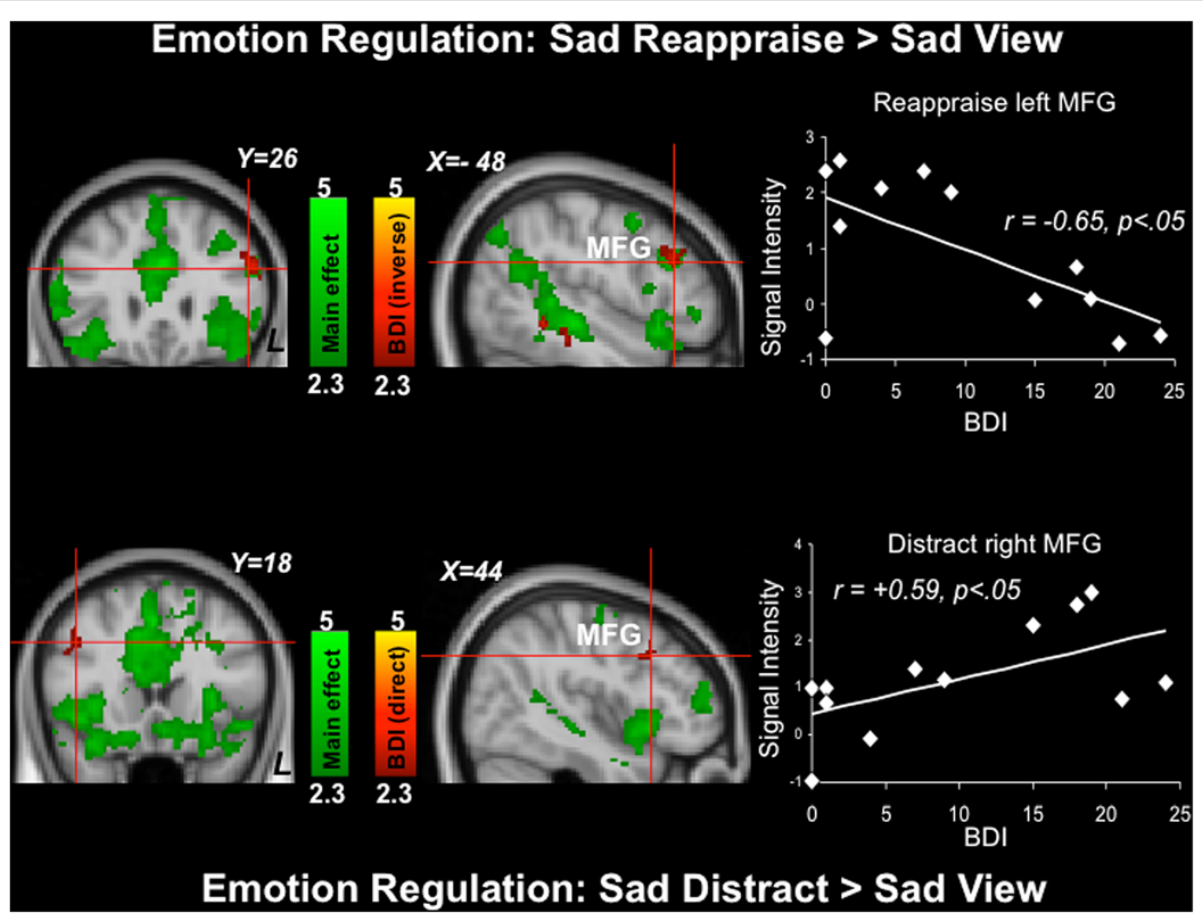

Figure 2 Emotion regulation results (Top: Sad-Reappraise > Sad View contrast; Bottom: Sad Distract>Sad View contrast). Green: Clusters significantly activated by the task. Red: Clusters significantly directly (top) and inversely (bottom) related to depression symptom severity. The scatterplots are provided for illustrative purposes and depict correlations between BDI scores with signal intensities of the red left (top) and right (bottom) midfrontal gyrus (MFG) clusters indicated by the crosshairs. 
Table 2 Cluster activations inversely associated with BDI scores during the Emotion Regulation task [Sad Reappraise > Sad View]

\begin{tabular}{|c|c|c|c|c|c|}
\hline \multirow[t]{2}{*}{ Region } & \multirow{2}{*}{$\begin{array}{l}\text { Size } \\
\left(\mathrm{mm}^{3}\right)\end{array}$} & \multirow{2}{*}{$\underset{\text { Max }}{Z}$} & \multicolumn{3}{|c|}{ MNI Coordinates } \\
\hline & & & $\mathrm{x}$ & $\mathrm{Y}$ & Z \\
\hline Frontal Gyrus (Left, Middle) & 744 & 2.98 & -50 & 24 & 26 \\
\hline Frontal Orbital Cortex (Right) & 296 & 2.82 & 42 & 32 & 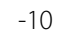 \\
\hline \multicolumn{6}{|l|}{ Frontal Pole } \\
\hline Right & 456 & 3.23 & 20 & 72 & 10 \\
\hline Left & 952 & 3.5 & -10 & 60 & 3 \\
\hline Parahippocampal Gyrus & 168 & 2.63 & -48 & -32 & -1 \\
\hline
\end{tabular}

(Left, Posterior)

Clusters are $>10$ voxels with $Z>2.3$.

scores were directly related to activation magnitudes in the right middle frontal gyrus $(\mathrm{Z} \mathrm{Max}=2.69)$, right precentral gyrus $(Z$ Max $=3.09)$, and left superior temporal gyrus $(\mathrm{Z} \mathrm{Max}=2.87)$ (see Table 4$)$.

\section{Resting state data}

Brain regions that covaried with the precuneus seed are depicted in (Figure 3 and Table 5). Intrinsic functional connectivity was observed between the precuneus seed and left postcentral gyrus $(Z \mathrm{Max}=5.19)$, right orbital frontal cortex $(\mathrm{Z} \mathrm{Max}=3.61)$, and bilateral temporal pole ( $\mathrm{Z} \mathrm{Max}=4.38$ for right and 3.43 for left). BDI scores directly predicted the strength of functional connectivity between the precuneus seed and left orbitofrontal cortex ( $\mathrm{ZMax}=4.42)$, left thalamus $(\mathrm{Z} \operatorname{Max}=3.58)$, right occipital cortex $(\mathrm{Z}$ Max $=3.96)$, bilateral parietal lobule $(\mathrm{Z}$ Max $=5.81$ for right and 3.91 for left), bilateral postcentral gyrus ( $\mathrm{Z} \mathrm{Max}=4.71$ for right and 3.89 for left), and bilateral precentral gyrus ( $\mathrm{Z}$ Max $=4.55$ for right and 4.59 for left) (see Table 6).

\section{In-scanner wheel of fortune affective ratings}

Average (SD) valence ratings on a scale of 1-6 after non-win trials was 2.87 (1.19) (1 depicted a very sad face and 6 depicted a neutral face). Average (SD) valence

Table 3 Clusters showing significant activation during the Emotion Regulation task [Sad Distract $>$ Sad View]

\begin{tabular}{lrrrrrr}
\hline Region & $\begin{array}{c}\text { Size } \\
\left(\mathbf{m m}^{\mathbf{3}}\right)\end{array}$ & $\begin{array}{c}\text { Z } \\
\text { Max }\end{array}$ & \multicolumn{3}{c}{$\begin{array}{c}\text { MNI } \\
\text { Coordinates }\end{array}$} \\
\cline { 5 - 8 } & & & X & Y & Z \\
\hline Cingulate Gyrus (Right, Posterior) & 295512 & 4.54 & 0 & -48 & 12 \\
Occipital Cortex (Right, Lateral, Superior) & 424 & 3.39 & 56 & -62 & 20 \\
Precentral Gyrus (Left) & 672 & 2.78 & -56 & 2 & 10 \\
Supramarginal Gyrus (Right, Posterior) & 3336 & 3.63 & 62 & -42 & 24 \\
Temporal Fusiform Cortex (Left, Posterior) & 248 & 2.97 & -32 & -26 & -28 \\
Temporal Gyrus (Right, Superior) & 2936 & 3.21 & 48 & -26 & -4 \\
\hline
\end{tabular}

Clusters are $>10$ voxels with $Z>2.3$.
Table 4 Cluster activations directly associated with BDI scores during the Emotion Regulation task [Sad Distract $>$ Sad View]

\begin{tabular}{lrrrrr}
\hline Region & $\begin{array}{c}\text { Size } \\
\left(\mathbf{m m}^{\mathbf{3}}\right)\end{array}$ & Z Z & \multicolumn{3}{c}{ MNI Coordinates } \\
\cline { 5 - 7 } & & & $\mathbf{X}$ & Y & Z \\
\hline Frontal Gyrus (Right, Middle) & 288 & 2.69 & 44 & 18 & 38 \\
Precentral Gyrus (Right) & 400 & 3.09 & 6 & -30 & 76 \\
Temporal Gyrus (Left, Superior) & 192 & 2.87 & -64 & -22 & 0 \\
\hline Clusters are $>10$ voxels with Z> 2.3. & & & & &
\end{tabular}

after win trials was 4.94 (0.86) (1 depicted a neutral face and 6 depicted a very happy face). BDI scores were not significantly correlated with valence ratings after win trials, $r=-0.44, p=0.15$ or after non-win trials, $r=-0.03$, $p=0.93$.

\section{Wheel of fortune imaging data}

Consistent with published approaches [22,35], analysis for each phase of the WOF task contrasted monetary and control trials.

\section{Reward selection phase}

The top of Figure 4 depicts results during reward selection (see also Table 7). Areas that showed activation included dorsal paracingulate gyrus ( $\mathrm{Z} \mathrm{Max}=3.45)$, anterior cingulate gyrus $(\mathrm{Z} \operatorname{Max}=2.97)$, and right frontal orbital cortex $(\mathrm{Z}$ Max $=3.15)$. BDI scores did not predict activation magnitudes in any region during this phase of the task.

\section{Reward anticipation phase}

The bottom of Figure 4 depicts results during reward anticipation (see also Table 8). Areas that showed activation included bilateral nucleus accumbens ( $\mathrm{Z}$ Max $=3.44$ for right and 2.79 for left,), right putamen ( $\mathrm{Z}$ Max = $2.67)$, and right thalamus $(\mathrm{Z} \mathrm{Max}=2.8)$. BDI scores did not predict activation magnitudes in any regions during this phase of the task.

\section{Reward outcome phase}

The top of Figure 5 depicts results during win outcomes (see also Table 9). Areas that showed activation included the left putamen $(\mathrm{Z}$ Max $=3.1)$, right thalamus ( $\mathrm{Z}$ Max $=2.89)$, and right amygdala $(Z \max =3.02)$. BDI scores did not predict activation magnitudes in any canonical reward regions during win outcomes. The bottom of Figure 5 depicts average results during non-win outcomes (see also Table 10). Areas that showed activation included the left paracingulate gyrus $(Z \max =3.61)$, left putamen $(Z$ Max $=2.80)$, and left thalamus $(Z$ Max $=$ 2.96). BDI scores were directly related to activation during non-win outcomes in the left paracingulate gyrus $(Z$ 


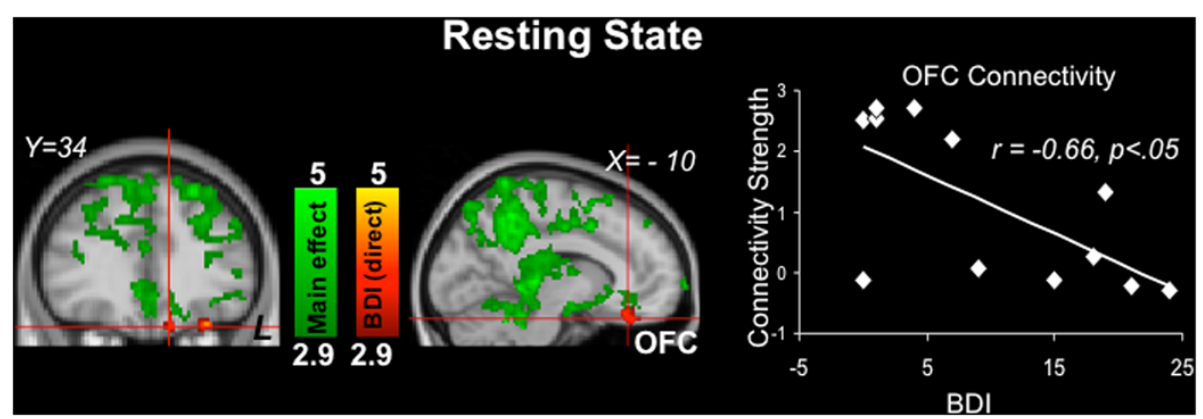

Figure 3 Resting state results. Green: Clusters with timecourses that significantly correlated with the timecourse of the precuneus seed region. Red: Clusters significantly directly related to depression symptom severity (there were no clusters with significant inverse associations with depression symptom severity). The scatterplot is provided for illustrative purposes and depicts the correlation of BDI scores with connectivity strength between the precuneus seed and the red orbital frontal cortex (OFC) cluster indicated by the crosshair.

$\operatorname{Max}=2.62)$ and the right caudate nucleus $(\mathrm{Z} \operatorname{Max}=$ 2.96) (See Table 11).

\section{Discussion}

The goal of the present study was to use fMRI to investigate linkages between patterns of brain activation and connectivity and subclinical levels of depressive symptoms. Emotion regulation, resting state, and reward processing paradigms were used that have been shown to differentiate MDD and control samples and to be related to core features of MDD. During the ER task,

Table 5 Clusters showing significant connectivity with the precuneus seed region during the resting state scan

\begin{tabular}{|c|c|c|c|c|c|}
\hline \multirow[t]{2}{*}{ Region } & \multirow[t]{2}{*}{$\begin{array}{l}\text { Size } \\
\left(\mathrm{mm}^{3}\right)\end{array}$} & \multirow[t]{2}{*}{$\underset{\operatorname{Max}}{Z}$} & \multicolumn{3}{|c|}{$\begin{array}{r}\mathrm{MN} \\
\text { Coordinates }\end{array}$} \\
\hline & & & $\mathrm{x}$ & $\mathrm{Y}$ & $\mathbf{Z}$ \\
\hline Frontal Gyrus, pars opercularis (Left, Inferior) & 24 & 3.79 & -58 & 14 & 28 \\
\hline Frontal Orbital Cortex (Right) & 21 & 3.61 & 44 & 16 & -10 \\
\hline \multicolumn{6}{|l|}{ Frontal Pole } \\
\hline Right & 157 & 3.75 & 20 & 66 & -4 \\
\hline Left & 83 & 3.73 & -32 & 36 & -10 \\
\hline Insular Cortex (Left) & 32 & 3.64 & -32 & 14 & 6 \\
\hline Occipital Cortex (Right, Lateral, Superior) & 117 & 4.64 & 42 & -74 & 18 \\
\hline Occipital Fusiform Gyrus (Right) & 215 & 4.49 & 20 & -80 & -18 \\
\hline Occipital Pole (Left) & 29 & 4.69 & -42 & -92 & 4 \\
\hline Planum Temporale (Right) & 28 & 3.57 & 56 & -26 & 8 \\
\hline Postcentral Gyrus (Left) & 59 & 5.19 & -48 & -20 & 54 \\
\hline Temporal Fusiform Cortex (Left, Posterior) & 20 & 4.11 & -68 & -26 & -20 \\
\hline Temporal Gyrus (Right, Middle, Posterior) & 158 & 3.9 & 60 & -20 & -12 \\
\hline $\begin{array}{l}\text { Temporal Gyrus, temporooccipital part } \\
\text { (Right, Inferior) }\end{array}$ & 61 & 3.79 & 46 & -56 & -12 \\
\hline \multicolumn{6}{|l|}{ Temporal Pole } \\
\hline Right & 34 & 4.38 & 48 & 6 & -26 \\
\hline Left & 42 & 3.43 & -44 & 10 & -18 \\
\hline
\end{tabular}

Clusters are $>10$ voxels with $z>2.9$. participants with higher BDI scores reported significantly poorer self-reported ER success. This pattern is consistent with the findings of Beauregard and colleagues [11], who found that individuals with MDD

Table 6 Clusters directly associated with BDI scores during the resting state scan

\begin{tabular}{|c|c|c|c|c|c|}
\hline \multirow[t]{2}{*}{ Region } & \multirow[t]{2}{*}{$\begin{array}{c}\text { Size } \\
\left(\mathrm{mm}^{3}\right)\end{array}$} & \multirow[t]{2}{*}{$\begin{array}{c}Z \\
\text { Max }\end{array}$} & \multicolumn{3}{|c|}{$\begin{array}{c}\text { MNI } \\
\text { Coordinates }\end{array}$} \\
\hline & & & $\mathrm{x}$ & $\mathrm{Y}$ & Z \\
\hline Cingulate Gyrus (Left, Posterior) & 37 & 3.56 & -4 & -20 & 42 \\
\hline Frontal Gyrus (Right, Superior) & 40 & 3.96 & 14 & 2 & 66 \\
\hline Frontal Orbital Cortex (Left) & 79 & 4.42 & -30 & 34 & -22 \\
\hline \multicolumn{6}{|l|}{ Frontal Pole } \\
\hline Left & 73 & 4.13 & -8 & 40 & -26 \\
\hline Right & 98 & 4.27 & 38 & 38 & 8 \\
\hline Lingual Gyrus (Right) & 28 & 3.53 & 30 & -46 & -4 \\
\hline Occipital Cortex (Left, Lateral, Inferior) & 27 & 3.55 & -38 & -74 & -2 \\
\hline Occipital Cortex (Lateral, Superior) & 25 & 3.53 & 42 & -74 & 16 \\
\hline Left & 23 & 3.25 & -24 & -78 & 24 \\
\hline Right & 51 & 3.56 & 42 & -58 & 36 \\
\hline Occipital Cortex (Right, Lateral, Superior) & 208 & 3.96 & 20 & -76 & 38 \\
\hline Occipital Pole (Right) & 29 & 3.76 & 12 & -90 & 20 \\
\hline \multicolumn{6}{|l|}{ Parietal Lobule (Superior) } \\
\hline Left & 28 & 3.91 & -32 & -54 & 70 \\
\hline Right & 781 & 5.81 & 26 & -46 & 60 \\
\hline Planum Temporale (Left) & 77 & 4.56 & -60 & -14 & 2 \\
\hline \multicolumn{6}{|l|}{ Postcentral Gyrus } \\
\hline Left & 46 & 3.89 & -48 & -18 & 34 \\
\hline Right & 529 & 4.71 & 48 & -20 & 52 \\
\hline \multicolumn{6}{|l|}{ Precentral Gyrus } \\
\hline Left & 174 & 4.59 & -58 & 2 & 18 \\
\hline Right & 285 & 4.55 & 30 & -10 & 46 \\
\hline Thalamus (Left) & 23 & 3.58 & -12 & -32 & 4 \\
\hline
\end{tabular}

Clusters are $>10$ voxels with $z>2.9$. 


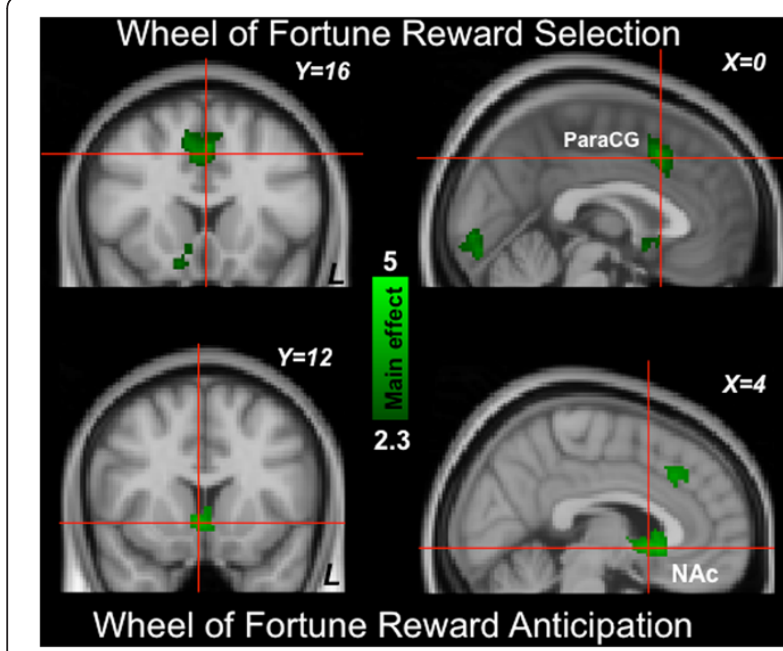

Figure 4 Wheel of Fortune results (Top: reward selection; Bottom: reward anticipation). Green: Clusters significantly activated by the task. No regions were significantly related to depression symptom severity. ParaCG: Paracingulate gyrus; NAc: nucleus accumbens.

reported relatively greater difficulty down-regulating feelings of sadness. Consistent with reports from nonclinical samples [6], group-level activation during reappraisal of sad stimuli was evident in the right middle frontal gyrus and bilateral temporal gyrus. Depressive symptom severity was related to activation magnitudes in right middle frontal gyrus during emotion regulation, though the laterality and direction of this finding was contingent on the type of ER strategy: left middle frontal gyrus activation was inversely related to activation during reappraisal, whereas right middle frontal gyrus activation was

Table 7 Clusters showing significant activation during the selection phase of the Wheel of Fortune task

\begin{tabular}{lrrrrrr}
\hline Region & $\begin{array}{c}\text { Size } \\
\text { ( } \mathbf{m m}^{\mathbf{3}} \mathbf{)}\end{array}$ & $\begin{array}{c}\text { Z } \\
\text { Max }\end{array}$ & \multicolumn{3}{c}{$\begin{array}{c}\text { MNI } \\
\text { Coordinates }\end{array}$} \\
\cline { 3 - 7 } & & & X & Y & Z \\
\hline Cingulate Gyrus (Left, Anterior) & 192 & 2.97 & -6 & 34 & 18 \\
Frontal Orbital Cortex (Right) & 1264 & 3.15 & 16 & 10 & -14 \\
Frontal Pole (Right) & 272 & 2.57 & 40 & 50 & 0 \\
Occipital Cortex (Left, Lateral, Inferior) & 160 & 2.99 & -42 & -72 & -18 \\
Occipital Cortex (Right, Lateral, Superior) & 25000 & 3.81 & -18 & -60 & 68 \\
Occipital Fusiform Gyrus (Right) & 24440 & 3.75 & -22 & -88 & -14 \\
Paracingulate Gyrus (Right) & 5288 & 3.45 & 0 & 16 & 44 \\
Parietal Lobule (Left, Superior) & 272 & 2.78 & -20 & -54 & 54 \\
Subcallosal Cortex (Left) & 488 & 2.78 & -4 & 14 & -10 \\
Supramarginal Gyrus (Left Anterior) & 616 & 3 & -52 & -28 & 48 \\
Temporal Gyrus, temporooccipital & 208 & 2.67 & 48 & -58 & -2 \\
(Right, Middle) & & & & &
\end{tabular}

Clusters are $>10$ voxels with $Z>2.3$.
Table 8 Clusters showing significant activation during the anticipation phase of the Wheel of Fortune task

\begin{tabular}{lrrrrrr}
\hline Region & Size & Z & \multicolumn{4}{c}{ MNI Coordinates } \\
\cline { 6 - 7 } & $\left(\mathbf{m m}^{\mathbf{3}}\right)$ & Max & X & Y & Z \\
\hline Accumbens & & & & & & \\
$\quad$ Right & 3560 & 3.44 & 6 & 18 & -4 \\
$\quad$ Left & 496 & 2.79 & -12 & 8 & -12 \\
Lingual Gyrus (Right) & 344 & 2.68 & 12 & -90 & -4 \\
Occipital Cortex (Left, Lateral, Inferior) & 1160 & 2.98 & -34 & -80 & 2 \\
Occipital Cortex (Lateral, Superior) & & & & & \\
$\quad$ Right & 2632 & 3.54 & 36 & -84 & 12 \\
$\quad$ Left & 536 & 3 & -24 & -60 & 34 \\
Occipital Pole (Left) & 416 & 2.75 & -2 & -92 & -8 \\
Paracingulate Gyrus (Right) & 1288 & 2.99 & 2 & 26 & 38 \\
Parietal Lobule (Superior) & & & & & \\
$\quad$ Right & 1296 & 2.83 & 18 & -58 & 62 \\
$\quad$ Left & 392 & 2.78 & -22 & -48 & 48 \\
Putamen (Right) & 104 & 2.67 & 16 & 14 & -12 \\
Thalamus (Right) & 360 & 2.8 & 12 & -20 & 18 \\
\hline
\end{tabular}

Clusters are $>10$ voxels with $Z>2.3$.

directly related to activation during distraction. These findings are consistent with neuroimaging data indicating that MDD is characterized by increased right middle frontal activation during reappraisal [6]. More broadly, these results suggest that brain activation during ER is related to the magnitude of subclinical levels of depressive symptoms.

During the resting state scan, intrinsic connectivity was observed between the precuneus seed region and the temporal cortex and anterior cingulate, similar to findings previously reported in nonclinical samples [15]. There was a significant positive correlation between depression symptom severity and the strength of functional connectivity between the precuneus seed region and the thalamus and left orbital frontal cortex. Given that MDD is characterized by increased DMN connectivity both during emotional processing paradigms and at rest $[18,19]$, the present findings suggest that increased DMN connectivity may characterize individuals with increasing levels of subclinical depressive symptoms, possibly reflecting heightened self-referential activities and ruminative cognitions.

During all phases of the reward processing task, participants demonstrated frontostriatal brain activation patterns similar to published reports from nonclinical samples, including dorsal paracingulate cortex activation during reward selection, striatal activation during reward anticipation, and medial prefrontal activation during reward outcomes [25,35]. Depressive symptom severity was not related to activation magnitudes in canonical 


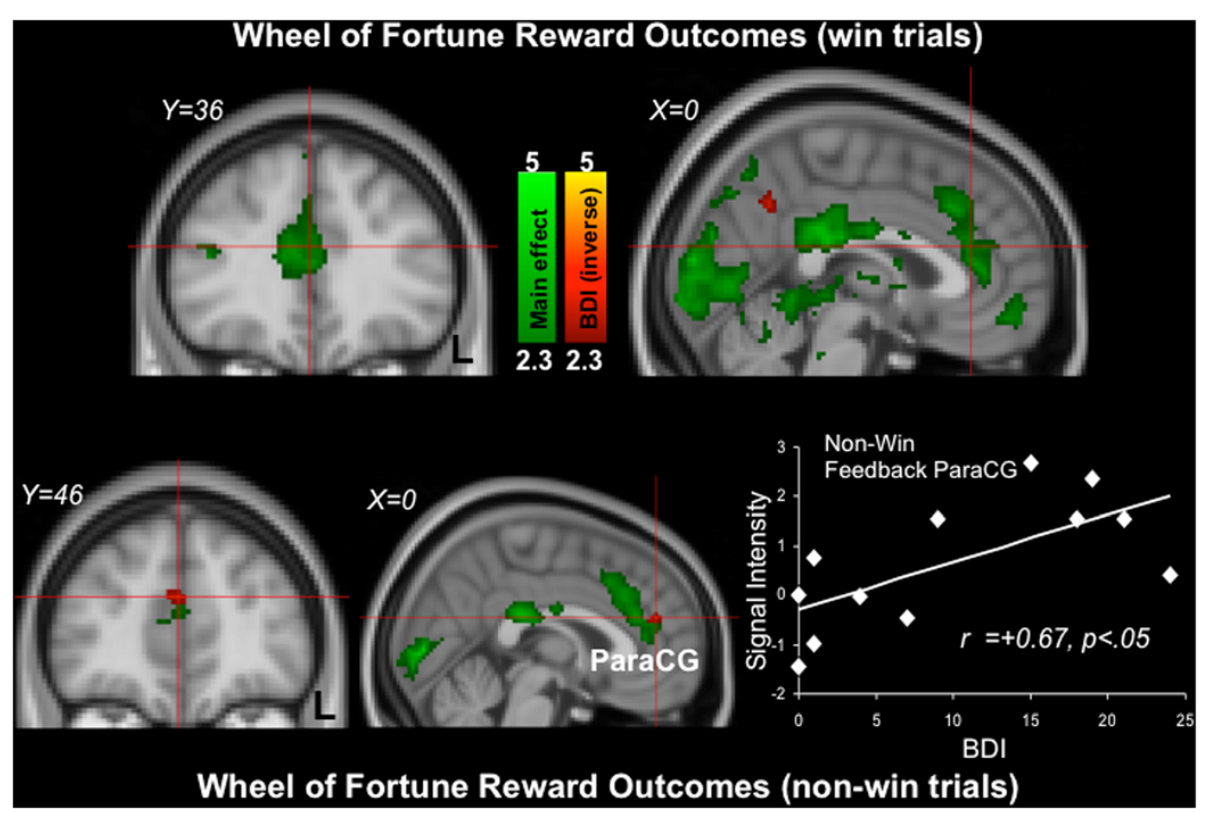

Figure 5 Wheel of Fortune results (Top: win outcomes; Bottom: non-win outcomes). Green: Clusters significantly activated by the task. Red: Clusters significantly inversely related to depression symptom severity. The scatterplot is provided for illustrative purposes and depicts correlations between signal intensity of the red paracingulate gyrus (ParaCG) cluster indicated by the crosshair with BDI scores.

reward regions during reward selection, reward anticipation, or win outcomes. However, depressive symptom severity was directly related to paracingulate gyrus activation during non-win outcomes. This pattern of findings is noteworthy when considered alongside the results of Pizzagalli and colleagues [23], who found greater differences between MDD and control samples during reward outcomes than during reward anticipation. There is also evidence that pediatric MDD is characterized by altered neural mechanisms of reward anticipation following reward outcomes [48], suggesting that neural responses to reward outcomes may impact future experiences with rewards. More generally, this pattern of results suggests that altered neural correlates of non-win reward outcome may be a more sensitive marker of subclinical depressive symptoms than neural responses during reward selection, reward anticipation or win outcomes.

The present study has a number of methodological limitations that should be considered when interpreting results. The relatively small sample and inclusion of only females suggests the need for replication in larger and more heterogeneous samples. Monthly variability in mood symptoms tied to menstrual phase and use of hormone contraceptives were not assessed, nor was participant handedness ${ }^{1}$. Finally, brain imaging analyses were conducted at uncorrected thresholds to protect against Type II errors in this pilot study, and future studies with larger samples will allow for replication with corrected statistical thresholds.
Table 9 Clusters showing significant activation during the outcome phase on win trials of the Wheel of Fortune task

\begin{tabular}{lrrrrrr}
\hline Region & $\begin{array}{c}\text { Size } \\
\left(\mathbf{m m}^{\mathbf{3}}\right)\end{array}$ & $\begin{array}{c}\text { Z } \\
\text { Max }\end{array}$ & \multicolumn{3}{c}{$\begin{array}{c}\text { MNI } \\
\text { Coordinates }\end{array}$} \\
\cline { 5 - 8 } & & & $\mathbf{X}$ & Y & $\mathbf{Z}$ \\
\hline Amygdala (Right) & 280 & 3.02 & 10 & -4 & -16 \\
Central Opercular Cortex (Right) & 104 & 2.68 & 46 & -16 & 16 \\
Cingulate Gyrus (Left, Anterior) & 10160 & 3.61 & -6 & 40 & 10 \\
Cingulate Gyrus (Right, Posterior) & 7144 & 4.19 & 2 & -30 & 24 \\
Frontal Gyrus (Left, Middle) & 408 & 2.94 & -50 & 18 & 36 \\
Frontal Gyrus (Right, Superior) & 88 & 2.76 & 24 & 2 & 62 \\
Frontal Gyrus, pars triangularis & 1400 & 3.06 & -42 & 34 & 16
\end{tabular}

(Left, Inferior)

Frontal Orbital Cortex

$\begin{array}{lrrrrr}\text { Right } & 664 & 2.83 & 18 & 14 & -16 \\ \text { Left } & 64 & 2.47 & -24 & 14 & -16\end{array}$

Frontal Pole

Right

Left

Insular Cortex (Left)

Occipital Cortex (Left, Lateral, Superior)

Occipital Cortex (Right, Lateral, Inferior)

Occipital Fusiform Gyrus (Left)

Parietal Lobule (Right, Superior)

Putamen (Left)

$\begin{array}{lllll}144 & 2.88 & 34 & 44 & 6\end{array}$

$\begin{array}{lllll}480 & 2.99 & -42 & 40 & 28\end{array}$

$\begin{array}{lllll}976 & 3.33 & -38 & 14 & -14\end{array}$

$\begin{array}{lllll}352 & 2.67 & -36 & -80 & 26\end{array}$

$\begin{array}{lllll}304 & 2.77 & 46 & -76 & 12\end{array}$

$\begin{array}{lllll}82544 & 4.49 & -20 & -88 & -8\end{array}$

$\begin{array}{lllll}11824 & 3.67 & 30 & -54 & 52\end{array}$

$\begin{array}{lllll}2472 & 3.1 & -22 & 2 & 8\end{array}$

Thalamus (Right)

$\begin{array}{lllll}704 & 2.89 & 20 & -16 & 14\end{array}$

Clusters are $>10$ voxels with $Z>2.3$. 
Table 10 Clusters showing significant activation during the outcome phase on non-win trials of the Wheel of Fortune task

\begin{tabular}{|c|c|c|c|c|c|}
\hline \multirow[t]{2}{*}{ Region } & \multirow[t]{2}{*}{$\begin{array}{l}\text { Size } \\
\left(\mathrm{mm}^{3}\right)\end{array}$} & \multirow[t]{2}{*}{$\underset{\operatorname{Max}}{\mathrm{Z}}$} & \multicolumn{3}{|c|}{$\begin{array}{c}\text { MNI } \\
\text { Coordinates }\end{array}$} \\
\hline & & & $x$ & $\mathrm{Y}$ & Z \\
\hline Cingulate Gyrus (Posterior) & 3128 & 3.8 & 0 & -30 & 24 \\
\hline Cuneal Cortex & 64 & 2.46 & 0 & -84 & 20 \\
\hline Frontal Gyrus (Left, Middle) & 1120 & 3.12 & -50 & 14 & 38 \\
\hline Frontal Gyrus (Left, Superior) & 344 & 2.9 & -8 & 16 & 60 \\
\hline Frontal Medial Cortex (Right) & 176 & 2.57 & 4 & 54 & -8 \\
\hline Frontal Operculum Cortex (Left) & 72 & 2.6 & -46 & 10 & 0 \\
\hline \multicolumn{6}{|l|}{ Frontal Orbital Cortex } \\
\hline Right & 304 & 2.82 & 38 & 18 & -16 \\
\hline Left & 120 & 2.92 & -24 & 24 & -4 \\
\hline Frontal Pole (Left) & 168 & 2.69 & -16 & 52 & 40 \\
\hline Hippocampus (Right) & 408 & 2.85 & 22 & -28 & -6 \\
\hline Insular Cortex (Left) & 1800 & 3.14 & -36 & 14 & -14 \\
\hline Intracalcarine Cortex & 27536 & 4.12 & 0 & -86 & 2 \\
\hline Lingual Gyrus (Right) & 216 & 2.61 & 8 & -56 & -6 \\
\hline Occipital Cortex (Right, Lateral, Superior) & 136 & 2.93 & 26 & -60 & 34 \\
\hline Occipital Fusiform Gyrus (Right) & 104 & 2.55 & 30 & -68 & -18 \\
\hline Paracingulate Gyrus (Left) & 8912 & 3.61 & -6 & 24 & 34 \\
\hline Parietal Lobule (Left, Superior) & 7128 & 3.25 & -32 & -46 & 40 \\
\hline Parietal Lobule (Right) & 872 & 2.86 & 26 & -52 & 42 \\
\hline Postcentral Gyrus (Left) & 216 & 2.66 & -54 & -24 & 42 \\
\hline Putamen (Left) & 616 & 2.8 & -22 & 8 & 4 \\
\hline Supramarginal Gyrus (Left, Anterior) & 1136 & 3.14 & -48 & -36 & 44 \\
\hline Temporal Gyrus (Left, Middle, Posterior) & 352 & 2.88 & -58 & -34 & -4 \\
\hline $\begin{array}{l}\text { Temporal Occipital Fusiform Cortex } \\
\text { (Right) }\end{array}$ & 104 & 2.54 & 36 & -56 & -8 \\
\hline Temporal Pole (Left) & 80 & 2.62 & -54 & 14 & -6 \\
\hline Thalamus (Left) & 1944 & 2.96 & -10 & -22 & 4 \\
\hline
\end{tabular}

Clusters are $>10$ voxels with $Z>2.3$.

\section{Conclusions}

Despite these limitations, this study suggests that subclinical depressive symptom severity is selectively related to regional brain activation patterns in key emotion regulation and reward processing regions and to DMN connectivity implicated in MDD. Although the three domains addressed here (i.e., emotion regulation, resting state connectivity, and reward processing) were assessed via separate tasks, there is clear conceptual overlap between these three domains. For example, prefrontal cortical recruitment is anomalous in MDD during regulation of rewarding stimuli [10] and DMN activity in MDD is abnormal during emotion regulation [18]. In this regard, the present findings may represent a conservative estimate of the effects of subclinical depressive
Table 11 Clusters inversely associated with BDI scores during the outcome phase of the Wheel of Fortune task

\begin{tabular}{|c|c|c|c|c|c|}
\hline \multirow[t]{2}{*}{ Region } & \multirow[t]{2}{*}{$\begin{array}{l}\text { Size } \\
\left(\mathrm{mm}^{3}\right)\end{array}$} & \multirow[t]{2}{*}{$\begin{array}{c}\text { Z } \\
\operatorname{Max}\end{array}$} & \multicolumn{3}{|c|}{$\begin{array}{c}\text { MNI } \\
\text { Coordinates }\end{array}$} \\
\hline & & & $\mathrm{X}$ & $\mathrm{Y}$ & Z \\
\hline Angular Gyrus (Left) & 520 & 2.73 & -38 & -56 & 18 \\
\hline Caudate (Right) & 80 & 2.57 & 6 & 10 & -2 \\
\hline Cingulate Gyrus (Right, Posterior) & 432 & 2.82 & 6 & -34 & 44 \\
\hline \multicolumn{6}{|l|}{ Frontal Gyrus (Middle) } \\
\hline Right & 248 & 3.04 & 28 & 18 & 46 \\
\hline Left & 152 & 2.58 & -30 & 26 & 48 \\
\hline \multicolumn{6}{|l|}{ Frontal Gyrus (Superior) } \\
\hline Right & 184 & 2.65 & 20 & 28 & 44 \\
\hline Left & 144 & 2.56 & -6 & 56 & 24 \\
\hline $\begin{array}{l}\text { Frontal Gyrus, pars opercularis } \\
\text { (Right, Inferior) }\end{array}$ & 72 & 2.53 & 52 & 16 & 28 \\
\hline Frontal Pole (Left) & 1008 & 2.93 & -18 & 56 & 22 \\
\hline Occipital Cortex (Right, Lateral, Superior) & 232 & 2.6 & 38 & -66 & 36 \\
\hline Paracingulate Gyrus (Left) & 200 & 2.62 & -4 & 44 & 22 \\
\hline Postcentral Gyrus (Left) & 80 & 2.65 & -48 & -16 & 32 \\
\hline Precentral Gyrus (Left) & 736 & 2.9 & -16 & -16 & 68 \\
\hline Precuneous Cortex & 64 & 2.62 & 0 & -58 & 38 \\
\hline Temporal Gyrus (Left, Middle, Posterior) & 288 & 2.96 & -58 & -24 & -12 \\
\hline $\begin{array}{l}\text { Temporal Gyrus, temporooccipital part } \\
\text { (Right, Middle) }\end{array}$ & 600 & 2.9 & 64 & -40 & -10 \\
\hline
\end{tabular}

Clusters are $>10$ voxels with $Z>2.3$.

symptoms on neural responses during tasks that simultaneously address these constructs.

Given that individuals with subclinical levels of depressive symptoms are at heightened risk for developing MDD [1,2], these results suggest candidate neural markers that may reflect trait vulnerability to MDD. Because MDD represents a highly prevalent, chronic, and costly disorder, identification of at risk individuals may provide critical windows for preventative interventions that represents a public health priority [49]. Future research using longitudinal and high-risk designs will be needed to more fully evaluate etiologic questions about MDD, MDD risk, and MDD resilience.

\section{Endnotes}

${ }^{a}$ We thank an anonymous reviewer for raising these points.

\section{Abbreviations}

BDI: Beck Depression Inventory-II; BOLD: Blood Oxygen Level Dependent; DMN: Default Mode Network; ER: Emotion Regulation; fMRI: Functional magnetic resonance imaging; GLM: General linear model; MDD: Major Depressive Disorder; MNI: Montreal Neurological Institute; WOF: Wheel of Fortune; SCID: Structured Clinical Interview for DSM-IV Patient Version.

\section{Competing interests}

The authors declare that they have no competing interests. 


\section{Authors' contributions}

JNF, MJS, and GSD conceived of and conducted the study and drafted the manuscript. RVK, BF, JM, JB, and CP participated in study design and data analysis and helped to draft the manuscript. All authors read and approved the final manuscript.

\section{Acknowledgements}

This research was supported by a Duke-UNC Brain Imaging and Analysis Center Post-Baccalaureate fellowship to J. Felder and by a UNC Research Council Award to G. Dichter. M. Smoski was supported by NIMH T32MH070448, a NARSAD Young Investigator award, and a career development award from Duke University Medical Center, NICHD K12 HD043446. G. Dichter was supported by NARSAD Young Investigator awards and by K23 MH081285. F. McClernon was supported by K23DA017261. The funding sources had no further role in study design, in the collection, analysis and interpretation of data; in the writing of the report; and in the decision to submit the paper for publication.

The authors would like to thank Todd Harshbarger and Syam Gadde for assistance with image analysis, Prue Cuper for SCID supervision, Dr. Nan-kuei Chen and Dr. James Voyvodic for helpful discussions regarding analysis of resting state data, MRI technologists Susan Music, Natalie Goutkin, and Luke Pool for assistance with data acquisition, and Dr. Allen Song for assistance with various aspects of this research. Assistance for this study was also provided by the Neuroimaging Core of the UNC Carolina Institute for Developmental Disabilities (P30 HD03110).

Publication of this article was funded by the University of Colorado Boulder Libraries Open Access Fund.

\section{Author details}

${ }^{1}$ Carolina Institute for Developmental Disabilities, University of North Carolina at Chapel Hill School of Medicine, CB\# 3366, 101 Manning Drive, Chapel Hill, NC 27599-7160, USA. ²Department of Psychiatry and Behavioral Sciences,

Duke University Medical Center, Durham, NC, USA. ${ }^{3}$ Department of Psychiatry, University of North Carolina at Chapel Hill School of Medicine, Chapel Hill, NC, USA. ${ }^{4}$ Duke-UNC Brain Imaging and Analysis Center, Duke University Medical Center, Durham, NC, USA. ${ }^{5}$ Department of Psychology and Neuroscience, University of Colorado Boulder, UCB 345, Boulder, CO 80309-0345, USA.

Received: 5 January 2012 Accepted: 24 August 2012

Published: 21 September 2012

\section{References}

1. Cuijpers P, Smit F: Subclinical depression: a clinically relevant condition? Tijdschr Psychiatr 2008, 50(8):519-528.

2. Brown GW, Bifulco A, Harris T, Bridge L: Life stress, chronic subclinical symptoms and vulnerability to clinical depression. J Affect Disord 1986, 11(1):1-19.

3. Kendler KS, Walters E, Truett KR, Heath AC, Neale MC, Martin NG, Eaves LJ: Sources of individual differences in depressive symptoms: analysis of two samples of twins and their families. Am J Psychiatry 1994, 151(11):1605-1614

4. Kendall PC, Butcher JN, Holmbeck GN: Conceptual and Methodological Issues in Research. In Handbook of Research Methods in Clinical Psychology (2nd ed.). New York: John Wiley \& Sons, Inc.; 1999

5. Mednick SA, McNeil TF: Current methodology in research on the etiology of schizophrenia: serious difficulties which suggest the use of the highrisk-group method. Psychol Bull 1968, 70(6):681-693.

6. Johnstone $\mathrm{T}$, van Reekum $\mathrm{CM}$, Urry HL, Kalin NH, Davidson RJ: Failure to regulate: counterproductive recruitment of top-down prefrontalsubcortical circuitry in major depression. J Neurosci 2007 27(33):8877-8884

7. Wager TD, Davidson ML, Hughes BL, Lindquist MA, Ochsner KN: Prefrontalsubcortical pathways mediating successful emotion regulation. Neuron 2008, 59(6):1037-1050.

8. Kim SH, Hamann S: Neural correlates of positive and negative emotion regulation. J Cogn Neurosci 2007, 19(5):776-798.

9. Light SN, Heller AS, Johnstone T, Kolden GG, Peterson MJ, Kalin NH, Davidson RJ: Reduced right ventrolateral prefrontal cortex activity while inhibiting positive affect is associated with improvement in hedonic capacity after 8 weeks of antidepressant treatment in major depressive disorder. Biol Psychiatry 2011, 70(10):962-968.

10. Heller AS, Johnstone T, Shackman AJ, Light SN, Peterson MJ, Kolden GG, Kalin NH, Davidson RJ: Reduced capacity to sustain positive emotion in major depression reflects diminished maintenance of fronto-striatal brain activation. Proc Natl Acad Sci U S A 2009, 106(52):22445-22450.

11. Beauregard $M$, Paquette $V$, Levesque J: Dysfunction in the neural circuitry of emotional self-regulation in major depressive disorder. Neuroreport 2006, 17(8):843-846.

12. Kanske P, Heissler J, Schonfelder S, Wessa M: Neural correlates of emotion regulation deficits in remitted depression: The influence of regulation strategy, habitual regulation use, and emotional valence. Neuroimage 2012, 61(3):686-693.

13. Raichle ME, MacLeod AM, Snyder AZ, Powers WJ, Gusnard DA, Shulman GL: A default mode of brain function. Proc Natl Acad Sci U S A 2001, 98(2):676-682.

14. Raichle ME: The restless brain. Brain Connect 2011, 1(1):3-12.

15. Fransson P: Spontaneous low-frequency BOLD signal fluctuations: an fMRI investigation of the resting-state default mode of brain function hypothesis. Hum Brain Mapp 2005, 26(1):15-29.

16. Greicius MD, Flores BH, Menon V, Glover GH, Solvason HB, Kenna $H$, Reiss $A L$, Schatzberg AF: Resting-state functional connectivity in major depression: abnormally increased contributions from subgenual cingulate cortex and thalamus. Biol Psychiatry 2007, 62(5):429-437.

17. Buckner RL, Andrews-Hanna JR, Schacter DL: The brain's default network: anatomy, function, and relevance to disease. Ann N Y Acad Sci 2008, 1124:1-38.

18. Sheline YI, Barch DM, Price JL, Rundle MM, Vaishnavi SN, Snyder AZ, Mintun MA, Wang S, Coalson RS, Raichle ME: The default mode network and self-referential processes in depression. Proc Natl Acad Sci U S A 2009, 106(6):1942-1947.

19. Sheline YI, Price JL, Yan Z, Mintun MA: Resting-state functional MRI in depression unmasks increased connectivity between networks via the dorsal nexus. Proc Natl Acad Sci U S A 2010, 107(24):11020-11025.

20. Zhou Y, Yu C, Zheng H, Liu Y, Song M, Qin W, Li K, Jiang T: Increased neural resources recruitment in the intrinsic organization in major depression. J Affect Disord 2010, 121(3):220-230.

21. Dichter GS, Damiano CA, Allen JA: Reward circuitry dysfunction in psychiatric and neurodevelopmental disorders and genetic syndromes: animal models and clinical findings. Journal of Neurodevelopmental Disorders 2012, 4

22. Smoski MJ, Felder J, Bizzell J, Green SR, Ernst M, Lynch TR, Dichter GS: fMRI of alterations in reward selection, anticipation, and feedback in major depressive disorder. J Affect Disord 2009, 118(1-3):69-78.

23. Pizzagalli DA, Holmes AJ, Dillon DG, Goetz EL, Birk JL, Bogdan R, Dougherty DD, losifescu DV, Rauch SL, Fava M: Reduced caudate and nucleus accumbens response to rewards in unmedicated individuals with major depressive disorder. Am J Psychiatry 2009, 166(6):702-710.

24. Knutson B, Bhanji JP, Cooney RE, Atlas LY, Gotlib IH: Neural responses to monetary incentives in major depression. Biol Psychiatry 2008, 63(7):686-692.

25. Kessler RC: Epidemiology of women and depression. J Affect Disord 2003, 74(1):5-13.

26. Kring $\mathrm{AM}$, Gordon $\mathrm{AH}$ : Sex differences in emotion: expression, experience, and physiology. J Pers Soc Psychol 1998, 74(3):686-703.

27. Spreckelmeyer KN, Krach S, Kohls G, Rademacher L, Irmak A, Konrad K, Kircher T, Grunder G: Anticipation of monetary and social reward differently activates mesolimbic brain structures in men and women. Soc Cogn Affect Neurosci 2009, 4(2):158-165.

28. Almeida JR, Kronhaus DM, Sibille EL, Langenecker SA, Versace A, Labarbara EJ, Phillips ML: Abnormal left-sided orbitomedial prefrontal cortical-amygdala connectivity during happy and fear face processing: a potential neural mechanism of female MDD. Front Psychiatry 2011, 2.69.

29. Keedwell PA, Chapman R, Christiansen K, Richardson H, Evans J, Jones DK: Cingulum white matter in young women at risk of depression: the effect of family history and anhedonia. Biol Psychiatry 2012, 72(4):296-302

30. Ray RD, Ochsner KN, Cooper JC, Robertson ER, Gabrieli JD, Gross JJ: Individual differences in trait rumination and the neural systems supporting cognitive reappraisal. Cogn Affect Behav Neurosci 2005, 5(2):156-168. 
31. Beck AT, Steer RA, Brown GK: Manual for Beck Depression Inventory-II. San Antonio, TX: Psychological Corporation; 1996.

32. First MB, Spitzer RL, Gibbon M, Williams JBW: Structured Clinical Interview for DSM-IV Axis I Disorders (SCID), Clinician Version; Administration Booklet. Washington, DC: American Psychiatric Press; 1996.

33. Wang L, McCarthy G, Song AW, LaBar KS: Amygdala activation to sad pictures during high-field (4 tesla) functional magnetic resonance imaging. Emotion 2005, 5(1):12-22.

34. Dichter GS, Felder JN, Smoski MJ: Affective context interferes with cognitive control in unipolar depression: An fMRI investigation. $J$ Affect Disord 2009, 114(1-3):131-142.

35. Ernst M, Nelson EE, McClure EB, Monk CS, Munson S, Eshel N, Zarahn E, Leibenluft E, Zametkin A, Towbin K, Blair J, Charney D, Pine DS: Choice selection and reward anticipation: an fMRI study. Neuropsychologia 2004, 42(12):1585-1597.

36. Voyvodic JT: Real-time fMRI paradigm control software for integrating stimulus presentation, Behavioral and physiological monitoring, and statistical analysis. Proc. Soc. Mag. Reson. Med. 1996, :1835. 15th Annual Meeting.

37. Smith SM, Jenkinson M, Woolrich MW, Beckmann CF, Behrens TEJ, Johansen-Berg H, Bannister PR, De Luca M, Drobnjak I, Flitney DE, Niazy RK, Saunders J, Vickers J, Zhang Y, DeStefano N, Brady JM, Matthews PM: Advances in functional and structural MR image analysis and implementation as FSL. Neurolmage 2004, 23(Suppl 1):S208-219.

38. Smith SM: Fast robust automated brain extraction. Human Brain Mapping 2002, 17(3):143-155.

39. Jenkinson M, Bannister P, Brady M, Smith S: Improved optimization for the robust and accurate linear registration and motion correction of brain images. Neuroimage 2002, 17(2):825-841.

40. Jenkinson M, Smith S: A global optimisation method for robust affine registration of brain images. Medical Image Analysis 2001, 5(2):143-156.

41. Woolrich MW, Ripley BD, Brady M, Smith SM: Temporal autocorrelation in univariate linear modeling of FMRI data. Neuroimage 2001, 14(6):1370-1386.

42. Vul E, Harris C, Winkielman P, Pashler H: Puzzlingly high correlations in fMRI studies of emotion, personality, and social cognition. Perspectives on Psychological Science 2009, 4(3):274-290

43. Lieberman MD, Cunningham WA: Type I and Type II error concerns in fMRI research: re-balancing the scale. Soc Cogn Affect Neurosci 2009, 4(4):423-428.

44. Thirion B, Pinel P, Meriaux S, Roche A, Dehaene S, Poline JB: Analysis of a large fMRI cohort: statistical and methodological issues for group analyses. Neuroimage 2007, 35(1):105-120

45. Dichter GS, Felder JN, Petty C, Bizzell J, Ernst M, Smoski MJ: The effects of psychotherapy on neural responses to rewards in major depression. Biol Psychiatry 2009, 66(9):886-897.

46. Dichter GS, Felder JN, Smoski MJ: The effects of brief behavioral activation therapy for depression on cognitive control in affective contexts: An fMRI investigation. J Affect Disord 2010, 126(1-2):236-244.

47. Dichter GS, Kozink RV, McClernon FJ, Smoski MJ: Remitted major depression is characterized by reward network hyperactivation during reward anticipation and hypoactivation during reward outcomes. $J$ Affect Disord 2012, 136(3):1126-1134.

48. Olino TM, McMakin DL, Dahl RE, Ryan ND, Silk JS, Birmaher B, Axelson DA, Forbes EE: "I won, but I'm not getting my hopes up": depression moderates the relationship of outcomes and reward anticipation Psychiatry Res 2011, 194(3):393-395.

49. Kessler RC: The costs of depression. Psychiatr Clin North Am 2012, $35(1): 1-14$

doi:10.1186/1471-244X-12-152

Cite this article as: Felder et al: Neural mechanisms of subclinical depressive symptoms in women: a pilot functional brain imaging study. BMC Psychiatry 2012 12:152.

\section{Submit your next manuscript to BioMed Central and take full advantage of:}

- Convenient online submission

- Thorough peer review

- No space constraints or color figure charges

- Immediate publication on acceptance

- Inclusion in PubMed, CAS, Scopus and Google Scholar

- Research which is freely available for redistribution

Submit your manuscript at www.biomedcentral.com/submit
C Biomed Central 